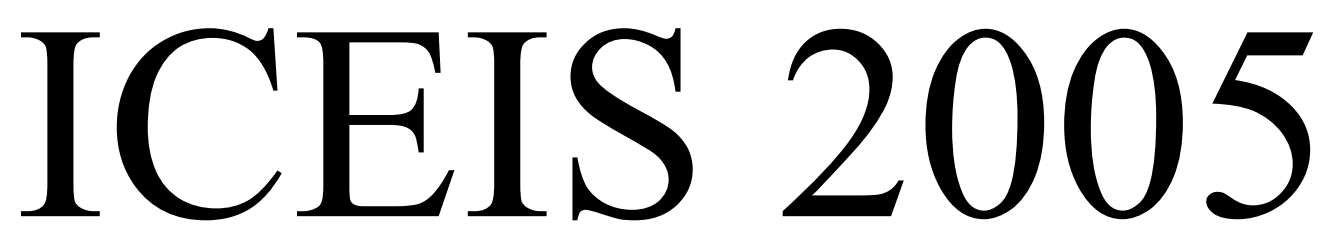

\author{
Proceedings of the \\ Seventh International Conference on \\ Enterprise Information Systems
}

Volume II

Miami, USA

May $25-28,2005$

Organized by

INSTICC - Institute for Systems and Technologies of Information, Control and Communication

Co-organized by

Florida International University

In cooperation with AAAI and ACM SIGMIS 


\title{
SYNTHESISE WEB QUERIES Search the Web by Examples
}

\author{
Vishv Malhotra, Sunanda Patro, David Johnson \\ School of Computing, Private Box 100, University of Tasmania, Hobart 7001 Australia \\ Email:vishv.malhotra@utas,.edu.au,spatro@postoffice.utas.edu.au,dgjohnso@utas.edu.au
}

Keywords: Boolean expression, web search query, web search engine, World Wide Web, Recall and precision of query

Abstract: An algorithm to synthesise a web search query from example documents is described. A user searching for information on the Web can use a rudimentary query to locate a set of potentially relevant documents. The user classifies the retrieved documents as being relevant or irrelevant to his or her needs. A query can be synthesised from these categorised documents to perform a definitive search with good recall and precision characteristics.

\section{INTRODUCTION}

The primary contribution of the paper is an algorithm that synthesises web search queries from examples of text documents categorised as relevant and irrelevant to the needs of a web searcher. The synthesised query would locate web links to resources relevant to the searcher as inferred from the examples. The three primary objectives for the algorithm are to ensure that the synthesised query has good recall; good precision; and not the least, is of a form and size acceptable to the intended search engine (Sebastiani 2002, Brin and Page 1998).

The rest of this paper is organised as follows. Section 2 gives an overview of the algorithm and uses examples to explain and provide a context for later sections. Sections 3, 4 and 5 describe the three main building block of the query synthesiser. Some of the issues arising from the algorithm and its current implementation are discussed in the final section.

\section{PRELIMINARIES}

A literal is an expression that is either a single term or its negation. In this paper, Boolean operator NOT (!) takes precedence over other Boolean operators. A minterm is a sequence of literals combined by Boolean operator AND $(\&)$. We would use Google convention to use AND $(\&)$ as lowest precedence and implied (not explicitly written) operator. A sequence of minterms combined by Boolean OR (|) operation is disjunctive normal form (DNF) of the Boolean expression. A maxterm is a sequence of literals combined by Boolean OR operations. A Boolean expression is in conjunctive normal form (CNF) if it is made of maxterms combined by operator AND. For further information about the Boolean expressions the reader may wish to see (Aho and Ullman, 1992) as an excellent reference.

A search query is suitably compacted representation of a DNF Boolean expression with at least one positive literal in each minterm. To help presentation of the algorithms in the later sections, we define a selection operation $\sigma$ : For a set of textual documents, $\mathrm{D}$, and a search query, $\mathrm{Q}$, expression $D \quad \sigma \quad Q$ will be used to denote a search by query $\mathrm{Q}$ over set $\mathrm{D}$. An operational interpretation of expression $D \sigma Q$ is as follows:

Case: $Q$ is term $\quad$ doc $\mid$ doc $\in D$ and term occurs in document doc $\}$

Case: $\mathrm{Q}$ is ! term $\quad$ doc $\mid$ doc $\in \mathrm{D} \&$ term does not occur in document doc

Case: $Q$ is $(R \quad \& \quad S) \quad(D \sigma R) \sigma S$

Case: $Q$ is $(R \mid S) \quad(D \sigma R) \cup(D \sigma S)$

The current implementation of the query synthesis algorithm requires the initial search query for retrieving example relevant and irrelevant documents to be a minterm comprising of positive literals. In this paper, the terms in the initial query minterm are deemed to occur in each document. A real document may not have query terms because search engines use, in addition to the text in the 
document, text near the links to the document in the other documents to index the linked document.

For a set, S, $|\mathrm{S}|$ denotes its cardinality - the number of elements in the set. For example, for a Boolean expression $B E$ and sets Relevant and Irrelevant we define its precision as: precision $(B E)$ $=\mid$ Relevant $\sigma B E|| \mid$ Irrelevant $\sigma B E \mid$. The case where denominator is 0 is handled by assigning a suitable large value as precision.

The five main steps in the query synthesis algorithm are:

i. Construct CNF (conjunctive normal form) Boolean expression that selects every document in set Relevant and rejects documents in set Irrelevant. This expression is typically long with many terms and Boolean operators to be efficient and effective search queries. See Section 3 for more details.

ii. Convert CNF Boolean expression into an equivalent DNF Boolean expression. Remove redundant minterms from the DNF expression. These minterms will be called p-minterms. Step 5 would modify the p-minterms to aid construction of an acceptable size search query. This will be discussed further in Section 3.1.

iii. Synthesise a Boolean expression Query by assembling a subset of minterms such that Query selects every document in set Relevant. Rewrite Query in a form suitable to the chosen web search engine. Details are in Section 4.

iv. If Query is acceptable to the search engine, stop. Boolean expression Query is the required search query. Otherwise,

v. Derive a set of minterms from p-minterms that supports synthesis of a better balanced search query. Go back to step 3 to synthesise a new candidate query. Section 5 deals with this issue.

\subsection{Examples}

To set a context and explain the steps in the algorithms, some example queries are given in Table
1. These queries were run over Google search engine to locate links to resources. A query with a single term (naïve query) was initially generated to retrieve documents for defining sets Relevant and Irrelevant. Only text documents are included in these sets. These sets are then used to synthesise a new query. The case of query regarding rainbow needs some further comments. In this case, the initial set Relevant had very few (three) documents for an effective synthesis of a query. The synthesised query (rainbow sky) did not perform well. However, the query synthesised from the documents retrieved by this query proved to be a good search query.

\section{CONSTRUCT CNF BOOLEAN EXPRESSION}

The search query synthesis begins with construction of a CNF Boolean expression that selects all relevant documents and rejects irrelevant documents. The Boolean expression can be constructed by repeatedly constructing maxterms to cover all relevant documents and reject many irrelevant documents. Sanchez, Triantaphyllou, Chen and Liao (2002) have reported an algorithm for maxterm construction by endeavouring to reject the irrelevant documents that have not been rejected by the previously constructed maxterms. Thus, after each successive stage, the conjunction of maxterms rejects more irrelevant documents.

We make two important changes to the reported algorithm to make it suitable for synthesising search queries.

i. We construct maxterms containing only positive literals. This conservative arrangement is appropriate because sets Relevant and Irrelevant do not characterise a closed world of documents.

Table 1: Examples of queries accessed using Google search engine. Type indicates the source of the query: naïve synthesised using the algorithm described in this paper, or a first attempt by a human.

\begin{tabular}{|l|l|l|l|l|}
\hline $\begin{array}{l}\text { Purpose } \\
\text { \& Type }\end{array}$ & Search query & $\begin{array}{l}\text { Query } \\
\text { size }\end{array}$ & $\begin{array}{l}\text { Relevant } \\
\text { accessed }\end{array}$ & $\begin{array}{l}\text { Irrelevant } \\
\text { accessed }\end{array}$ \\
\hline Information about radioactive element Radium. & 1 & 19 & 41 \\
\hline Naive & radium & 7 & 62 & 8 \\
\hline Synthetic & radium (((element / period) number)/((element / metal) uranium)) & 3 & 50 & 20 \\
\hline Human & radium element radioactive & 1 & 3 & 52 \\
\hline Information regarding rainbow that appear in sky after a rain. & 2 & 16 & 48 \\
\hline Naive & rainbow & 9 & 43 & 27 \\
\hline Synthetic & (rainbow sky) & 3 & 37 & 33 \\
\hline Synthetic & rainbow light (raindrop / higher/ copyright / solar / hand index dark)) & \\
\hline Human & (rainbow rain color) & & \\
\hline
\end{tabular}


ii. Each term (word in the example documents) is prioritised to reflect its potential as the next candidate term in the maxterm under construction.

\section{Definition}

The potential of a candidate term is defined by the number of new relevant and irrelevant documents it selects. The potential for term $t$ during construction of $(\mathrm{i}+1)^{\mathrm{st}}$ maxterm is computed as follows (maxterm $^{\mathrm{p}}$ is the partially constructed $(\mathrm{i}+1)^{\mathrm{st}}$ maxterm):

$\mathrm{TR}=$ Relevant $-\left(\right.$ Relevant $\sigma$ maxterm $\left.^{\mathrm{p}}\right)$;

$\mathrm{TR}_{\mathrm{t}}=\mathrm{TR} \sigma \mathrm{t}$

TIR $=$ Irrelevant $\sigma\left(\right.$ maxterm $_{1} \& \ldots \&$ maxterm $\left.\left._{\mathrm{i}}\right)\right)$;

$\mathrm{TIR}_{\mathrm{t}}=\mathrm{TIR} \sigma \mathrm{t}$;

$\operatorname{Potential}(\mathrm{t})=$

$$
\left(\left(\left|\mathrm{TR}_{\mathrm{t}}\right|\right)\left(|\mathrm{TIR}|-\left|\mathrm{TIR}_{\mathrm{t}}\right|\right)\right) /\left(\left(|\mathrm{TR}|-\left|\mathrm{TR}_{\mathrm{t}}\right|+1\right)\left(\left|\mathrm{TIR}_{\mathrm{t}}\right|+1\right)\right) .
$$

An algorithm for constructing CNF Boolean expression is shown in Figure 1. The algorithm terminates successfully when a conjunction of maxterms rejects every irrelevant document. The changes made to the algorithm could, in some rare cases, lead to a failed run of function Build_Maxterm. If all terms in a relevant document are also in an irrelevant document then it is not possible to find a term for a maxterm that would select the relevant document but would reject the irrelevant document. It is practical to remove these irrelevant documents from set Irrelevant. The price for this action is a small reduction in the precision of the synthesised query.

\subsection{Eliminate Boolean Expression Redundancies}

The Boolean expressions constructed by algorithm Build_CNF_BE frequently has too many terms for an effective and efficient processing by the web search engines. As an example of a constructed Boolean query consider the case of search for information about rainbow shown in Table 1 . Starting with query (rainbow sky) a set of 16 relevant and 48 irrelevant documents were identified. The Boolean expression for this example is: rainbow (raindrop / arc / prism / solar / term / bow / hand) (air / higher / band / design / contact / sunlight / american) (red / copyright / download / index) (light) (water / green / board / dark). Clearly, the query is not suitable for a Google search.

To eliminate some redundancy the synthesised CNF Boolean expression can be transformed into an equivalent DNF expression using the distribution property: $A(B / C) \equiv(A B) /(A C)$. The application

\section{Build_CNF_BE}

Input: Relevant, Irrelevant, Original Query.

Output: BoolExp

Description: BoolExp selects all documents in set Relevant and rejects virtually all documents in set Irrelevant.

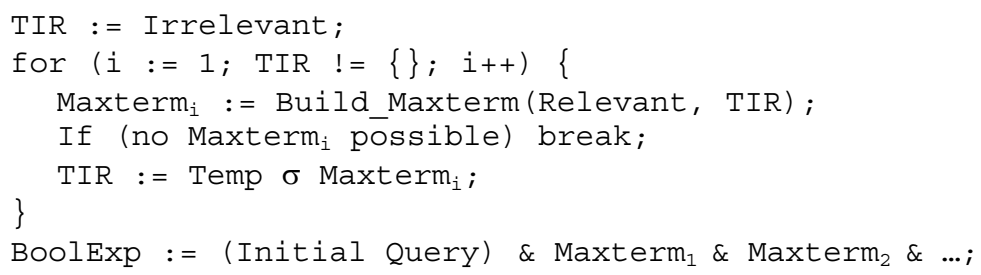

\section{Build_Maxterm}

Input: Relevant, TIR

Output: maxterm

Description: maxterm selects every document in set Relevant and rejects some document in set TIR

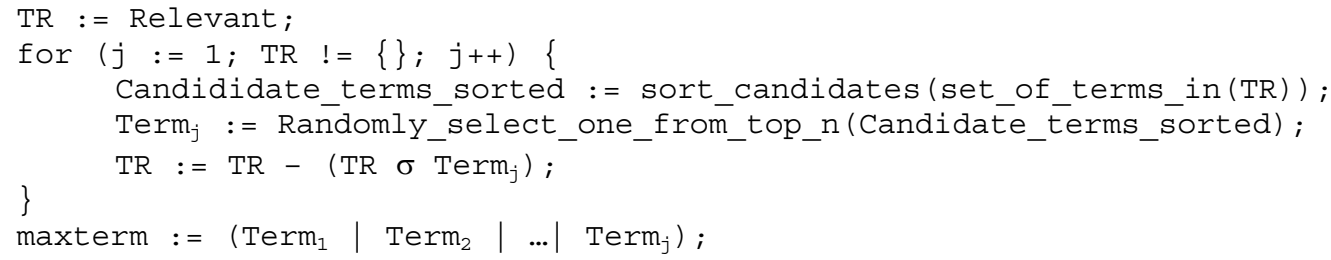

Figure 1: Algorithm to construct a Boolean expression that selects all relevant documents and reject irrelevant documents. 
of this rule would generate $7 \times 7 \times 4 \times 4=784$ minterms, each with 6 (or fewer) terms for the above example. Equivalence of two forms of the Boolean expression - the original CNF and the transformed DNF implies that each minterm would reject each document in set Irrelevant. The set of minterms in the DNF Boolean expression collectively select every document in set Relevant. However, an individual minterm may or may not select any document in set Relevant. Minterms that do not select any document in set Relevant are redundant and can be removed. All but 241 minterms of the original 748 minterms in DNF Boolean expression for the rainbow query were removed through an application of this rule.

We also remove those minterms from this set that select a proper subset of relevant documents selected by another minterm in the set. As a further step in redundancy reduction, terms are removed from minterms if their removal does not lead to selection of an irrelevant document. The minterms surviving these purges constitutes $p$-minterms ( $p$ signifies precise, perfect or preferred).

\section{SEARCH QUERY: MINIMUM MINTERM COVER FOR SET RELEVANT}

A minimal set of minterms that selects each document in set Relevant constitutes a search query. The set of p-minterms collectively selects all documents in set Relevant. At the same time, a document may be selected by several minterms. For example, the query concerning rainbow has 16 relevant documents, but has $241 \mathrm{p}$-minterms. No more than 16 minterms are needed to select 16 documents. As the minimisation problem is in NPComplete, we use a heuristic to construct a minterm cover for set Relevant.

A number of heuristics are possible to meet the goal of keeping the size of the query small while covering every document in set Relevant. The Search query synthesis heuristic we use, builds covering minterm set by selecting a minterm at a time from the set of available minterms. A greedy choice is made to select the minterm based on the number of new relevant documents selected by the minterm divided by the increase in the query size.

Apte, Damerau and Weiss (1994) have reported a more sophisticated heuristic for a similar problem. In their approach, an augmentation is either an addition of a new minterm to the query or is a substitution of a minterm in the query with another minterm. In our heuristic, we derive similar benefits by using a genetic algorithm approach. We construct the query several times by starting with a randomly selected first minterm. The query with the smallest number of terms is affirmed as the search query.

Given a set of minterms comprising a query it is useful to define function minimise to transform the set into a query acceptable to Google (or some other) search engine. We use the number of terms in this minimised query as its size. An exact syntax of an acceptable query for Google is not well defined. Thus, we use a simplistic algorithm to minimise the query representation. To construct a minimal query from a given set of minterms the function identifies a term that occurs most frequently in the query minterms. The set of minterms can be partitioned into two sets.

i. A set of minterms that do not contain the identified term.

ii. The set of minterms that contains the term. The term is factored out of these minterms by using equivalence: $\left(\begin{array}{ll}A & B\end{array}\right) \mid\left(\begin{array}{ll}A & C\end{array}\right) \equiv A \quad(B \mid C)$.

The minimisation function is then applied recursively to two sets to take advantage of other terms that can be used to reduce the query size. As an example consider the DNF expression: (radium element number) / (radium period number) / (radium element uranium) / (radium metal uranium). The expression has 12 terms. The number of terms can be reduced to 7 by the minimisation algorithm leading the following query: radium (((element / period) number) / ((element / metal) uranium)). Deeply nested expressions may not be accepted by some search engines. Consequently we would avoid synthesising such queries.

In our experiments we noticed only a few cases of queries with more than 10 terms when synthesised from p-minterms. Typical query is wellsized for the search engines. An oversize query for the rainbow example is: rainbow ((raindrop light) / (((higher red) / solar) water) / (bow copyright) / (term red) / (hand index dark)).

\section{FITTING QUERY TO SEARCH ENGINE}

If a synthesised query has too many terms it needs modifications to help synthesise acceptable queries. This issue will be discussed in this section.

Size of a query can be reduced to fit it to the constraints of a search engine. However, it would attract cost in terms of loss in its ability to select all relevant documents or not being able to avoid all irrelevant documents. If query size is reduced by reducing its ability to select all relevant documents, we need to perform supplementary searches to 


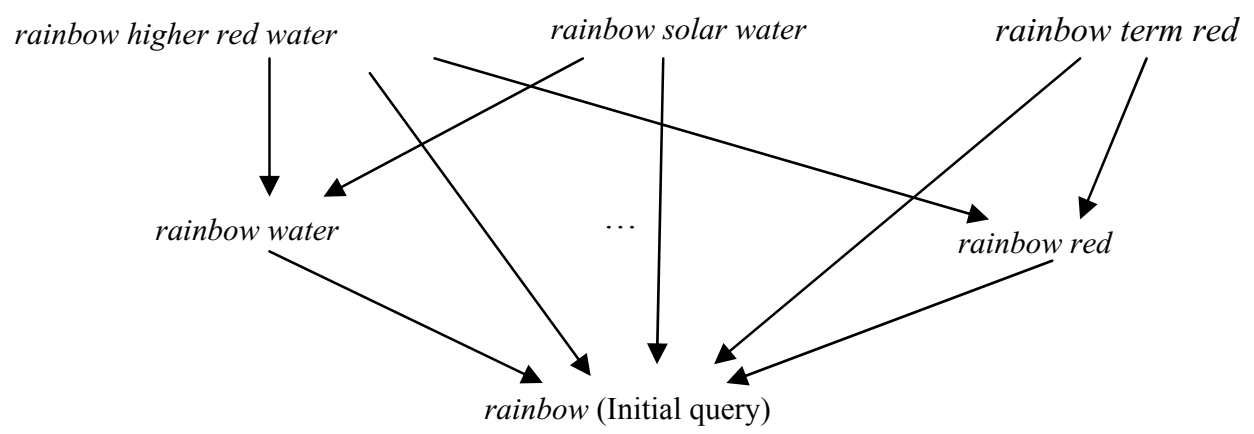

Figure 2: A part of lattice spawned by p-minterms for rainbow query.

account for the documents that we might miss. Human users tend to use this mode of search. If a query fails to return a satisfying collection of relevant links, the query is modified to seek other links of interest. In contrast to human-controlled interactive web session, the aim of a synthesised query is to make each search comprehensive by keeping the recall at its highest level and seek to reduce query size by sacrificing precision. Thus, we seek a query that meets the constraints of the search engine and selects every document in set Relevant. However, in addition it may select documents in set Irrelevant. The goal is to minimise the number of selected documents from the latter set.

For the oversized query for rainbow, the query can be made smaller in size by removing terms (for example) water, solar, higher or red. A term is removed by replacing it by Boolean constant true. The terms in the initial query are not available for removal as such a change may alter the membership of sets Relevant and Irrelevant. Each removal of term from a query affects its size differently. For example, the rainbow query will be one term smaller if one of the terms, water, higher or red is removed from it. It will be smaller by three terms if term solar is removed.

A removal of a term from a minterm may cause it to select a few irrelevant documents. Thus, there is a lowering in minterm's ability to reject irrelevant documents. As an approximation, we measure the precision of a query by the minimum precision of its composing minterms. Consequently, query obtained by removing term water has precision minimum \{precision(rainbow solar), precision(rainbow higher red) $\}$. On the other hand, if term solar is removed, it will be precision(rainbow water). The irrelevant documents are likely to have lower correlation among distributions of query terms. These observations lead us to a simple and effective method for reducing the query size by lowering their precision in a controlled way.

The method involves replacing two or more $\mathrm{p}$ minterms by a single minterm implied by each of the p-minterms. The replacing minterm selects all relevant documents selected by the replaced $\mathrm{p}$ minterms. It has fewer terms thus helping the synthesised query to be smaller in size. However, the query would have some lowering of its ability to reject all irrelevant documents.

Alternately, one could attempt to create smaller queries by defining minterms at lower precision values by removing one or more terms from $\mathrm{p}$ minterms. However, such an approach may generate a lot of minterms with only marginal advantage in reducing the query size. The method suggested in the previous paragraph is able to generate a well spread lattice of minterms (see Figure 2) to synthesise query near any required precision value.

A reduction in size of a synthesised but oversize query by removal of some terms from its minterms must compete against other queries that may be synthesised through similar concessions. Thus, the query synthesis is repeated whenever minterms have been affected by a transformation that alters their precisions.

The rest of this section contains a series of definitions that provide an outline of a method for constructing sets of minterms from p-minterms. The basic idea will be to give a method for deriving implied minterms from the given set of $\mathrm{p}$-minterms. Next we will define a set by selecting minterms meeting a stipulated precision property. The set will be called a set of good minterms. An important property of p-minterms that the set preserves is the ability to cover all relevant documents. Thus, using minterms in the set, one can synthesise a query at a precision value matching the precision of the minterms in the set. If the query turns out to be too 
large, the synthesis is repeated by constructing a new set of good minterms at the next lower precision level for which minterms can be found. The initial query guarantees that the repetitions will eventually terminate successfully; in the worst case we may have the initial query as the best query for the search.

\section{Definition}

Let $\mathrm{R}$ and $\mathrm{S}$ be two sets of minterms, $\operatorname{coalesce}(\mathrm{R}, \mathrm{S})$ is a set of minterms defined as follows:

coalesce $(\mathrm{R}, \mathrm{S})=\{\pi(\mathrm{r}, \mathrm{s}) \mid \mathrm{r} \in \mathrm{R}$ and $\mathrm{s} \in \mathrm{S}\}$,

where $\pi$ is defined as follows:

$\pi\left(\mathrm{m}_{1}, \mathrm{~m}_{2}\right)=\mathrm{m}$, where $\mathrm{m}$ is a minterm such that $\mathrm{m}_{1} \Rightarrow \mathrm{m}, \mathrm{m}_{2} \Rightarrow \mathrm{m}$, and for every minterm $\mathrm{m}$ '

Definition

$$
\left(\mathrm{m}_{1} \Rightarrow \mathrm{m}^{\prime} \Rightarrow \mathrm{m} ; \mathrm{m}_{2} \Rightarrow \mathrm{m}^{\prime} \Rightarrow \mathrm{m}\right) \Rightarrow\left(\mathrm{m}^{\prime} \equiv \mathrm{m}\right) \text {. }
$$

Let $\mathrm{M}$ be a set of p-minterms. We derive set of all useful minterms, $\mathrm{U}$ as follows: $\mathrm{U}=\mathrm{M}^{1} \cup \mathrm{M}^{2} \ldots$; where $\mathrm{M}^{1}=\mathrm{M}$ and $\mathrm{M}^{\mathrm{i}+1}=\operatorname{coalesce}\left(\mathrm{M}^{\mathrm{i}}, \mathrm{M}\right)$.

The finite size of set of terms makes it easy to see that for some finite $\mathrm{k}, \mathrm{M}^{\mathrm{k}}=\mathrm{M}^{\mathrm{k}+1}$. To each $\mathrm{u} \in \mathrm{U}$ we associate precision $(\mathrm{u})=\mid$ Relevant $\sigma \mathrm{u} \mid /$ $\mid$ Irrelevant $\sigma \mathrm{u} \mid$; where denominator is 0 , a large value is assigned as precision.

A set of minterms where each minterm meets a stipulated constraint precision value is a set of good minterms. These minterms can be used to synthesise query at the matching precision level. Formally,

\section{Definition}

$\operatorname{GoodMinterms}(\mathrm{U}, \mathrm{p})=\{\mathrm{u} \in \mathrm{U} \mid \operatorname{precision}(\mathrm{u}) \geq \mathrm{p} \&$ $\forall \mathrm{w} \in \mathrm{U}[(\mathrm{u} \Rightarrow \mathrm{w}) \Rightarrow((\operatorname{precision}(\mathrm{w})<\mathrm{p}) \mid(\mathrm{u} \equiv \mathrm{w}))]\}$

The precision values in set $U$ are discrete; by progressively lowering the value one can derive a query that meets the search engine constraints at the best precision value possible.

\section{CONCLUDING REMARKS}

The paper has described a method for constructing search query from a collection of relevant and irrelevant text documents. The method constructs the query by following a series of stages. Each stage is a greed-driven heuristic to certain goals. In our experiments, we found the greedy approach to be adequate; there is little need to seek optimised and perfect Boolean expressions as there are uncertainties outside our controls. For example, the small number of documents used to construct the query can only define a very imprecise image of documents and resources on the Web. Efforts spent on matching this imperfect image would not deliver proportionate rewards.
The method performs well when a reasonable collection of relevant and irrelevant documents is available. As the size of the collection increases, it becomes better image of the resources on the Web! Too small a size of the set does not help in synthesis of a good quality search query. A very large collection, on the other hand, requires additional processing effort. Particularly, it will require more human effort to classify the documents in the collection. The goal of the exercise is to reduce human effort. A set of about 10 relevant documents with 10 to 20 irrelevant documents are generally adequate.

The constructed queries include some unintuitive terms. However, the queries retrieve fresh links and are precise when used over the Web. An unintuitive term is one that web searchers are not likely use in queries. An unintuitive term is not a counterintuitive term.

The tests that we have conducted using the algorithm to this stage have shown very good improvements in the quality of links to resources in comparison with the links retrieved using naïve queries. Even in the areas well understood by a user, the synthesised queries have performed better than those constructed by humans. Some performance results are available in Patro and Malhotra (2005).

\section{REFERENCES}

Aho, A.V. and Ullman, J.D., 1992. Foundations of computer science, Computer Science Press, New York.

Apte, C., Damerau, F.J., and Weiss, S.M., 1994. Automated Learning of Decision Rules for Text Categorisation, ACM Transactions on Information Systems, 3(12). 233-251.

Brin, S. and Page, L., 1998. The anatomy of a large-scale hypertextual web search engine, J. Computer Networks and ISDN Systems, 30(1). 107-117.

Patro, S. and Malhotra V. 2005, Characteristics of the Boolean web search query: Estimating success from characteristics, http://eprints.comp.utas.edu.au:81/

Sanchez, S.N., Triantaphyllou, E., Chen, J. and Liao, T.W., 2002. An Incremental Learning Algorithm for Constructing Boolean Functions from Positive and Negative Examples, Computers and Operations Research 29(12). 1677-1700.

Sebastiani, F. 2002. Machine learning in automated text categorization, ACM Computing Surveys, 34(1): 1-47. 
Copyright (C INSTICC - Institute for Systems and Technologies of Information, Control and Communication

All rights reserved

Edited by Chi-Sheng Chen, Joaquim Filipe, Isabel Seruca and José Cordeiro

Printed in Portugal

ISBN: 972-8865-19-8

http://www.iceis.org

secretariat@iceis.org 